九州大学学術情報リポジトリ

Kyushu University Institutional Repository

\title{
Kinetic Analysis of Lysozyme-Inhibitor Complex Formation
}

Aso, Yoichi

Laboratory of Biochemistry, Faculty of Agriculture, Kyushu University

Koga, Daizo

Laboratory of Biochemistry, Faculty of Agriculture, Kyushu University

Mori, Fumio

Laboratory of Sericultural Chemistry, Faculty of Agriculture, Kyushu University

Hayashi, Katsuya

Laboratory of Sericultural Chemistry, Faculty of Agriculture, Kyushu University

他

https://doi.org/10.5109/22849

出版情報: 九州大学大学院農学研究院紀要. 18 (2)，pp. 89-106，1974-03. Kyushu University バージョン：

権利関係 : 


\title{
Kinetic Analysis of Lysozyme-Inhibitor Complex Formation
}

\author{
Yoichi Aso, Daizo Koga, Fumio Mori, Katsuya Hayashi \\ and Masaru Funatsu \\ Laboratory of Sericultural Chemistry, Laboratory of \\ Biochemistry, Faculty of Agriculture, \\ Kyushu University, Fukuoka
}

(Received December 28, 1973)

\begin{abstract}
It has been well recognized that the reaction rates in equilibrium steps such as enzymesubstrate complex formation or transformation of the complex in the enzymatic reaction can only be measured by special techniques such as temperature-jump method, because the reaction velocity of equilibrium step not accompanying the molecular change is generally large beyond the limit of adoption of usual kinetic methods. The authors reported previously that the enzyme-substrate complex formation in the system of the substrate and the enzyme-inhibitor complex had quite smaller reaction rate than expected one. When the substrate was added to the solution of enzyme-inhibitor complex which was previously formed, it was found that the exchange of inhibitor with the substrate took about $90 \mathrm{~min}$ for the completion of a new equilibrium state. This fact leads us to suggest that the rate constant of the step of the enzyme-substrate complex formation would be measured by usual kinetic method, if the experiment is designed so as to involve the exchange of inhibitor by the substrate in the reaction system.

Before measuring the rate constant of the enzyme-substrate complex formation based upon the above principle, it will be absolutely necessary to analyze the process of the enzyme-inhibitor complex formation to obtain the kinetic parameters of the system. The present article reports the results of kinetic analysis of the complex formation between lysozyme and the cationic detergent dimethylbenzylmyristylammonium chloride and some discussions on relevant subjects.
\end{abstract}

\section{INTRODUCTION}

An enzymatic reaction involves generally one or more equilibrium reaction steps. Kinetic analyses for enzymatic reactions have usually been discussed by the terms of the initial velocity and the Michaelis constant $\boldsymbol{K}_{m}$ based upon the steady-state kinetics. However, in such a treatment, the linearization approximation of nonlinear ordinary differential equations derived from the enzymatic reaction scheme has been known to make the characteristic behaviors of the enzymatic reaction obscure. For the analysis of dynamic aspect of enzymatic reaction- especially its time-course -, it is necessary first of all to have information about the kinetic constant of reaction steps which describes the reaction system.

Hayashi et al. (1968a, 1968b) have reported that the cationic detergent dimethylbenzylmyristylammonium chloride inhibited the activity of lysozyme competitively. The interaction between the detergent and lysozyme showed a 
considerably slow reaction rate toward the equilibrium state. When in this equilibrated system was mixed glycol chitin as the substrate, it was observed that the difference spectrum caused by the interaction between lysozyme and the detergent decreased slowly owing to the exchange of the detergent molecule bound on the enzyme by the added substrate. Generally, the reaction rate constant in enzyme-substrate complex formation can be measured directly using special technique such as temperature-jump method, because of its great reaction velocity. However, it is suggested from the above findings that the evaluation of the kinetic constants for the enzyme-substrate complex formation in such an exchanging system may be possible without direct measurement of the reaction rate. The equilibrium reaction of lysozyme with the detergent showed characteristic features, for example, the equilibrating rate is not proportional to the initial concentration of the detergent. It is interesting to see which reaction scheme explains kinetically such characteristic behavior of the complex formation. In this sense it is thought to be significant to establish the method to determine the rate constants of the equilibrium step in the enzymatic reaction.

The present paper deals mainly with the analysis of the interaction between lysozyme and dimethylbenzylmyristylammonium chloride on the basis of the optimization method.

\section{EXPERIMENTAL}

\section{Materials}

Dimethylbenzylmyristylammonium chloride (abbreviated by DBMA) supplied by the Kao-Soap Co. Ltd. was purified by recrystallization from the absolute ether and tetrahydrofuran according to the method of Duynstee and Grunwald (1959) with minor modifications. Lysozyme was purified by the method described previously (Hayashi et al., 1963) and other reagents used were of analytical grade.

\section{Methods}

\section{Time-course of lysozyme-inhibitor interaction (I)}

The time-course of change in the intensity in the difference spectrum at 296 $\mathrm{nm}\left(\Delta \mathrm{OD}_{i 96}\right)$ was observed according to the method described in a previous paper (Hayashi et al., 1968a). The initial concentration of lysozyme was fixed at 0.1 $\%$ and the initial concentrations of DBMA were varied from $0.05 \%$ to $5.0 \%$. The reaction mixture was kept at $30^{\circ} \mathrm{C}$ in $\mathbf{0 . 1} \mathrm{M}$ phosphate buffer, $\mathrm{pH}$ 5.6. The difference spectrum was measured by a Cary-14 Recording Spectrophotometer equipped with a Haake Thermostat type F.

\section{Time-course of lysoyzme-inhibitor interaction (II)}

Lysozyme and DBMA was mixed rapidly in the mixing chamber of a Hitachi Rapid-Scanning Spectrophotometer. The time dependence of the difference spectrum was observed by a Hitachi Memory-Scope. In the reference compartment, cell was filled with the reaction mixture which was mixed previously and equilibrated sufficiently. The reaction mixture was kept at $18^{\circ} \mathrm{C}$ in $0.1 \mathrm{M}$ citratephosphate buffer, pH 5.6. 


\section{Computation}

All computations were performed by FACOM 230-60 Digital Computer and some FACOM SSL in the Computer Center, Kyushu University.

\section{RESULTS}

\section{Time-course of lysozyme-inhibitor-interaction (I)}

Figs. 1,2 and 3 show the time-course of change in $A \mathrm{OD}_{296}$ caused by the mixing of lysozyme and DBMA. The reaction ratio, the ordinate, is defined in this paper as the ratio of $A \mathrm{OD}_{296}$ at each time to $\triangle \mathrm{OD}_{296}$ at the equilibrium state. The values of $\Delta \mathrm{OD}_{296}$ at the equilibrium state are shown in Fig. 4. In the high concentration region of DBMA, $\boldsymbol{A} \mathrm{OD}_{2.96}$ at the equilibrium state shows discontinuity. The time-course in the region below $0.1 \%$ of DBMA concentration was therefore mainly analyzed. Table 1 shows the reciprocal relaxation times calculated from the time-course of the interaction between lysozme and DBMA.

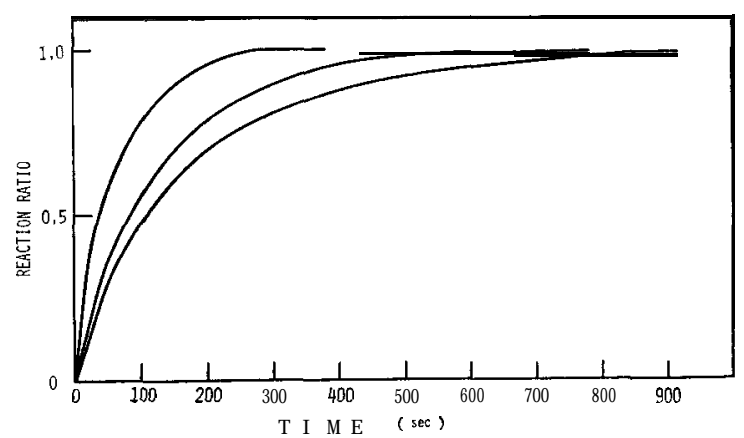

Fig. 1. The time dependence of $\Delta \mathrm{OD}_{296}$. The concentrations of lysozyme were fixed at $0.1 \%$ and of DBMA from top to bottom were $0.05 \%, 0.08 \%$ and $0.1 \%$.

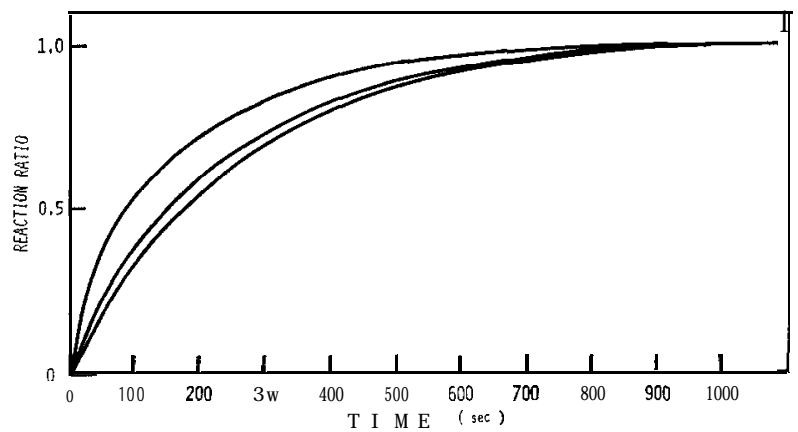

Fig. 2. The time dependence of $\triangle \mathrm{OD}_{296}$. The concentrations of lysozyme were fixed at $0.1 \%$ and of DBMA from top to bottom were $0.2 \%, 0.6 \%$ and $0.8 \%$. 


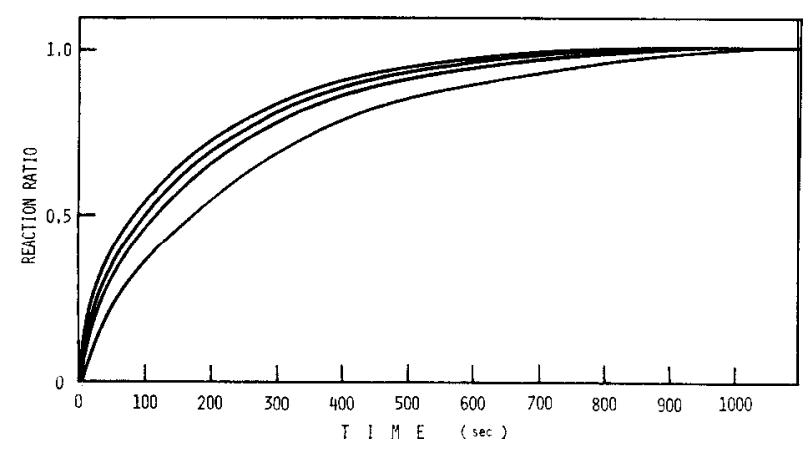

Fig. 3. The time dependence of $\triangle O D_{296}$. The concentrations of lysozyme were fixed at $0.1 \%$ and of DBMA from top to bottom were $2.0 \%, 4.0 \%, 5.0 \%$ and $1.0 \%$.

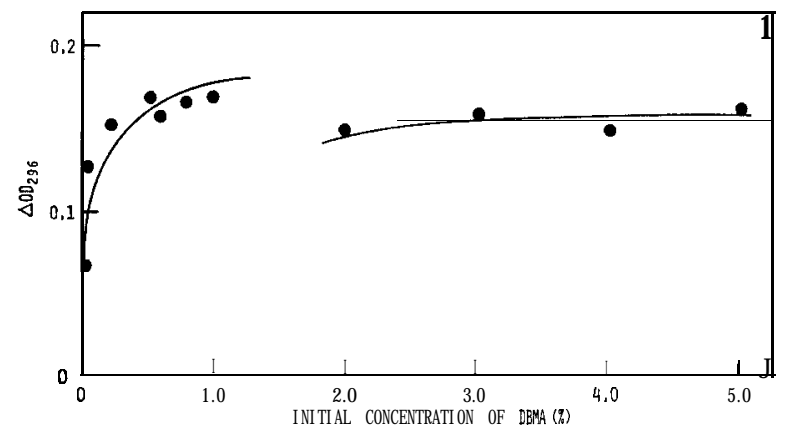

Fig. 4. The intensity of the difference spectrum at $296 \mathrm{~nm}$ in the equilibrium state as a function of the concentraition of DBMA.

Table 1. The reciprocal relaxation times for the binding of DBMA to lysozyme.

\begin{tabular}{c|c|c}
$\begin{array}{c}\text { Concentration } \\
\text { of } \begin{array}{c}\text { lysozyme } \\
\left(\times 10^{-4} \mathrm{M}\right)\end{array}\end{array}$ & $\begin{array}{c}\text { Concentration } \\
\text { of DBMA } \\
\left(\times 10^{-1} \mathrm{M}\right)\end{array}$ & $\begin{array}{c}\text { Reciprocal } \\
\text { relaxation time } \\
\left(\times 10^{-2} \mathrm{sec}^{-1}\right)\end{array}$ \\
\hline 0.680 & 0.0282 & 0.432 \\
0.680 & 0.0565 & 0.431 \\
0.680 & 0.1410 & 0.377 \\
0.680 & 0.1690 & 0.396 \\
0.680 & 0.2250 & 0.367 \\
0.680 & 0.5650 & 0.306 \\
0.680 & 0.8460 & 0.444 \\
0.680 & 1.130 & 0.459 \\
0.680 & 1.410 & \\
\hline
\end{tabular}

\section{Time-course of lysozyme - inhibitor interaction (II)}

The time-course of change in $\triangle \mathrm{OD}_{296}$ is shown in Fig. 5 . Since, in the reference cell, the mixture accomplished already the equilibrium state, the difference spectrum at an elongated reaction time should be disappeared. However, practically, the small bubbles in the mixing chamber disturbed sometimes the base-line of spectrum and the irregular shaped spectrum remained at an elongated reaction time. The height, $A \mathrm{OD}_{296}$, was measured from a line which was drawn 
in parallel with abscissa through the point on the base-line at $340 \mathrm{~nm}$, because the base-line at $340 \mathrm{~nm}$ was held invariantly during the reaction.

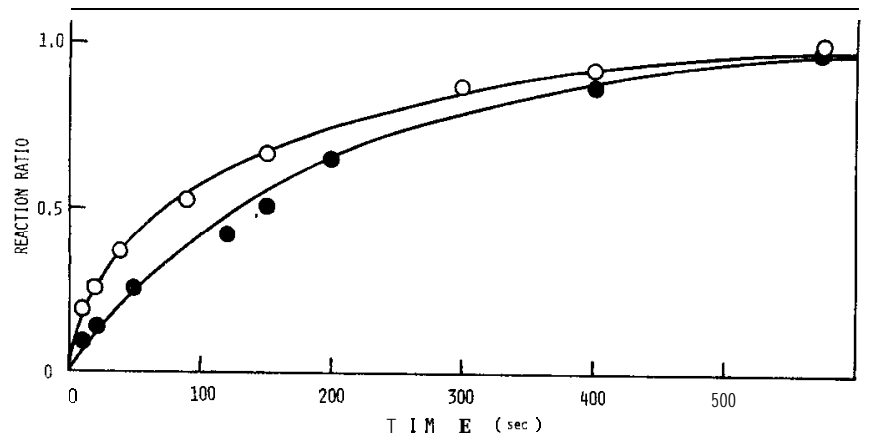

Fig. 5. The time dependence of $\triangle \mathrm{OD}_{296}$. The concentration of lysozyme was 0.1048 $\times 10^{-3} \mathrm{M}$. The concentrations of DBMA were $0.8474 \times 10^{-3} \mathrm{M}(0)$ and $0.14124 \times 10^{-2}$ $\mathrm{M}(\mathbf{O})$.

\section{THEORY}

\section{Interaction in Enzyme-Substrate-Inhibitor System}

The simplest equilibrium reaction process between enzyme, E, substrate, S, and inhibitor, I, can be expressed by the following scheme.

$$
\mathrm{EI} \underset{k_{1}}{\stackrel{k_{2}}{\rightleftarrows}} \mathrm{E}+\mathrm{I} \quad+\mathrm{S} \underset{k_{4}}{\stackrel{k_{3}}{\rightleftarrows}} \mathrm{ES} \stackrel{k_{5}}{\longrightarrow} \mathrm{P}
$$

where $\mathrm{P}$ is product, $k$, and $k_{2}$ are the rate constants for the enzyme-inhibitor complex formation and $k_{3}$ and $k_{4}$ are for the enzyme-substrate complex formation and $k_{3}$ the product formation. When the substrate is mixed in this equilibrated enzyme-inhibitor system, which is enclosed by the dotted line in equation (1), the time-course of change in the concentrations of the enzyme-substrate complex, $\mathrm{X}(\mathrm{r})$, and the enzyme-inhibitor complex, $\mathrm{Y}(\mathrm{t})$, may be written by the following simultaneous differential equations :

$$
\left\{\begin{array}{l}
\frac{d X(t)}{d t}=a X(t)+b Y(t)+c \\
\frac{d Y(t)}{d t}=d X(t)+e Y(t)+f
\end{array}\right.
$$

where

$$
\begin{aligned}
& a=-\left(k_{3} S_{0}+k_{4}+k_{5}\right), b=-k_{3} S_{0}, \\
& c=k_{3} S_{0} E_{0}, \quad d=-k_{1} I_{0}, \quad e=-\left(k_{1} I_{0}+k,\right), \\
& f=k_{1} I_{0} E_{0} .
\end{aligned}
$$

The $E_{0}, I_{0}$, and $S_{0}$ are the initial concentrations of enzyme, inhibitor and substrate, respectively, and it is assumed that $E_{0} \ll S_{0}$ and $E_{0} \ll I_{0}$. 
Thus, when the values of $k_{1}$ and $k_{2}$ for the enzyme-inhibitor interaction are evaluated, one can estimate the values of $k_{3}$ and $k_{4}$ by numerical computation.

\section{Interaction in Enzyme-Inhibitor System}

\section{Chemical relaxation method}

With the chemical relaxation method (Eigen and DeMaeyer,1963), the equilibrating rate of the reaction system may be represented by the following equation :

$$
\frac{d(\Delta X)}{d t}=-\left(\frac{1}{\tau}\right) \Delta X
$$

or

$$
\ln \frac{\Delta X}{(\Delta X)_{t=0}}=-\left(\frac{1}{\tau}\right) t
$$

where $A X$ is the deviation of chemical species from its equilibrium concentration and $\tau$ is the relaxation time.

(A) Single-step equilibrium reaction :

Let us represent the reaction system of enzyme, E, with inhibitor, I, by

$$
\mathrm{E}+\mathrm{I} \underset{k_{2}}{\stackrel{k_{1}}{\rightleftarrows}} \mathrm{EI} \text {. }
$$

In this reaction, the relation between the rate constants and the reciproca relaxation time can be expressed as

$$
1 / \tau=k_{2}+k_{1}\{(\bar{E})+(\bar{I})\}
$$

where $(\bar{E})$ and $(\bar{I})$ are the equilibrium concentrations of enzyme and inhibitor, respectively. With the plotting of the reciprocal relaxation time $v s$ the sum of the equilibrium concentrations, the rate constants $k_{1}$ and $k_{2}$ are calculated from the slope and the intercept of the curve. If the equilibrium constant is difficult to determine experimentally, one may use the iterative computation that need not require the known value of equilibrium constant on the estimation of rate constant. Considering that the equilibrium concentrations are calculated from the equilibrium constant and the latter is calculated in turn from the rate constants, one may use the following algorithm for the computation of equation (6).

a). Give the value of estimated equilibrium constant $K^{j}$. The superscript $\mathrm{j}$ means that it is the $\mathrm{j}$-th estimate, for example, the $K^{1}$ is the initially assumed value.

b). Calculate the equilibrium concentrations by the initial concentrations and the newly revised equilibrium constant.

c). Evaluate the rate constants $k_{1}^{j}$ and $k_{2}{ }^{j}$ by equation (6) with the least-squares approximation.

d). Give the $(j+1)$-th estimate of the equilibrium constant by the following relation.

$$
K^{j+1}=k_{1}{ }^{i} / k_{2}{ }^{j}
$$


e). Iterate the procedures from a) to d) until a given convergence condition is satisfied.

(B) Two-step equilibrium reaction :

The general expression of the relation between the relaxation times and the rate constants for multiple-step equilibrium reaction has been derived by Castellan (1963). In accordance with his representations, the reciprocal relaxation time is given as the root of the following characteristic equation:

$$
\begin{aligned}
& \left|r_{\alpha} g_{\alpha \beta}-\lambda \delta_{\alpha \beta}\right|=0 \\
& \lambda=1 / \tau_{\varepsilon} \quad(\varepsilon=1,2,3, \ldots \ldots) \\
& r_{\alpha}=\left(\frac{k_{\alpha}}{r_{\alpha}^{*}}\right) \prod_{j} \bar{a}_{j}^{-\nu_{j \alpha}}=\left(\frac{k_{-\alpha}}{\gamma_{\alpha}^{*}}\right) \prod_{j^{\prime}} \bar{a}_{j^{\prime}, y^{\prime} \alpha} \\
& g_{\alpha \beta}=\sum_{i=1}^{N} \nu_{i \alpha} \bar{\nu}_{i \beta}
\end{aligned}
$$

where $\delta_{\alpha \beta}$ is Kronecker's delta, $r_{\alpha}$ is the exchange rate of the a-th elementary reaction, $r_{\alpha}{ }^{*}$ is the activity coefficient, $\mathrm{j}$ and $\mathrm{j}^{\prime}$ represent $\mathrm{j}$-th reactant and the $j^{\prime}$-th product in the $\alpha$-th reaction, respectively, and $\bar{a}$ the equilibrium concentration of chemical species in the $\alpha$-th reaction and $\beta$-th reaction and $\bar{c}$ the corresponding equilibrium concentration. The $\nu$ represents the stoichiometric coefficient, and it has negative sign for the reactant and positive sign for the product.

Sharma and Leussing (1971) have employed the residue given by the following expression :

$$
\mathbf{R} \mathbf{i}=\left|r_{\alpha} g_{\alpha \beta}-\lambda_{i} \hat{\delta}_{\alpha \beta}\right|(i=1,2, \ldots N)
$$

where $R_{i}$ is the $\mathrm{i}$-th residue and $\lambda_{i}$ is the $\mathrm{i}$-th observative of the reciprocal relaxation time. The $R_{i}$ and the equilibrium concentration are in nonlinear relationship with the rate constants. Obtaining the analytical or numerical partial derivative of the residue with respect to the rate constants, therefore, one should use the nonlinear least-squares method. If the reaction scheme is reliable, one can theoretically estimate the rate constants without observing all kinds of relaxation times with this method.

Consider the following reaction scheme for the two-step equilibrium reaction :

$$
\mathrm{E}_{1} \underset{k_{2}}{\stackrel{k_{1}}{\rightleftarrows}} \mathrm{E}_{2}+\mathrm{I} \underset{k_{4}}{\stackrel{k_{3}}{\rightleftarrows}} \mathrm{E}_{2} \mathrm{I}
$$

where $\mathrm{E}_{2}$ represents one of the different conformational state (isomer and conformer) of the enzyme $\mathrm{E}$, The residue may be expressed by

$$
\mathbf{R}=\left|\begin{array}{lr}
k_{1}+k_{2}-\lambda & -k_{2} \\
-k_{3} \bar{I} & k_{3}\left(\overline{E_{2}}+\bar{I}\right)+k_{4}-\lambda
\end{array}\right|
$$

or

$$
R=\lambda^{2}-(\nu+\alpha) \lambda+(\alpha \nu-\delta \beta)
$$

where 


$$
\begin{aligned}
& \alpha=k_{1}+k_{2}, \quad \beta=-k_{2} \\
& \delta=-k_{3} \quad \bar{I}, \quad \nu=k_{3}\left(\bar{E}_{2}+\bar{I}\right)+k_{4}
\end{aligned}
$$

The $\bar{E}_{2}$ and $\bar{I}$ are the equilibrium concentrations of $\mathrm{E}_{2}$ and I, respectively.

\section{Direct curve-fitting method}

Solving numerically the ordinary differential equations describing the equilibrium reaction, one may compare the calculated reaction time-course with the expeirmentally obtained one. Then, by the appropriate optimization methods, the optimum rate constants may be directly estimated so as to fit them with those in the real reaction. The Fletcher-Reeves' method (FR method) may be applied as one of the optimization methods. The algorithm of the FR method can be summarized as follows:

a). Give $P_{1}$ as the initially assumed value of the parameters (initial guess) to be estimated.

b). Compute $S_{1}=-g_{1}=-\nabla^{T} F\left(P_{3}\right)$, where $\nabla^{T} F\left(P_{1}\right)$ is the partial derivative of the objective function (performance index) $F$ with respect to the parameter $P$,. The subscript 1 represents the calculated value at first iteration.

c). Select the value of $\alpha_{j}$ which minimizes the function $F\left(\boldsymbol{P}_{j}+\alpha_{j} \boldsymbol{S}_{j}\right)$ by some linear search.

d). Give the $(\mathrm{j}+1)$-th estimate ef the parameter $P$ by the following expression:

$$
P_{j+1}=P_{j}+\alpha_{j} S_{j}
$$

e). Revise the value of $\mathrm{S}$ in the next iteration by the equation

$$
\begin{aligned}
S_{j+1} & =-g_{j+1}+\frac{g_{j+1}^{T} \cdot g_{j+1}}{g_{j}^{T} \cdot g_{j}} S_{j}(j \neq \mathrm{m}+1) \\
g_{j} & =\nabla^{T} F\left(P_{j}\right)
\end{aligned}
$$

where $\mathrm{m}$ is the number of the parameters, and when $\mathrm{j}=\mathrm{m}+\mathrm{l}, S_{m+1}=-g_{m+1}$. f). Iterate the above steps until a given convergence condition is satisfied.

(A) Single-step equilibrium reaction :

Consider the equilibrium reaction represented by equation (5). The simultaneous differential equation describing the system may be written as

$$
\left\{\begin{array}{l}
\frac{d X(t)}{d t}=k, Z(t) Y(t)-k_{2} X(t) \\
\frac{d Y(t)}{d t}=-k, Z(t) Y(t)+k_{2} X(t) \\
\frac{d Z(t)}{d t}=-k_{1} Z(t) Y(t)+k_{2} X(t)
\end{array}\right.
$$

where $X(t)$ is the concentration of the enzyme-inhibtor complex at time $t, Y(t)$ is the concentration of the enzyme, and $Z(t)$ the concentration of inhibitor. Then the concentration of the enzyme-inhibitor complex may be expressed by

$$
X(t)=k_{1} \int_{0}^{t} Z(\tau) Y(\tau) d \tau-k_{2} \int_{0}^{t} X(\tau) d \tau
$$


The relative residue of the calculated value for the observed value is defined as

$$
R_{i}=\left(X_{i} / X_{e q}-\Delta O D_{i} / \Delta O D_{e q}\right) /\left(\Delta O D_{i} / \Delta O D_{e q}\right)
$$

where $X_{i}$ and $A O D$, are values of $\mathrm{X}(\mathrm{t})$ and $A O D$ at i-th data point, respectively, and $X_{e q}$ and $\triangle O D_{e q}$ are values of $X(t)$ and $A O D$ at the equilibrium state, respectively. Then, the optimization may be stated as follows:

$$
F=\sum_{i=1}^{n} R_{i}^{2} \longrightarrow \min
$$

where $F$ is the objective function and $\mathrm{n}$ is the number of data points. The partial derivatives of the objective function $F$ with respect to the rate constants are

$$
\frac{\partial F}{\partial k_{j}}=2 \sum_{i=1}^{n} R_{i} \frac{\partial R_{i}}{\partial k_{j}} \quad(\mathrm{i}=1,2, \ldots \mathrm{n} ; \mathrm{j}=1,2, \ldots, \mathrm{m})
$$

where $\mathrm{m}$ is the number of rate constants and

$$
\begin{aligned}
& \partial R_{i} \\
& \partial k_{j}
\end{aligned}=\left(\left(\partial X_{i} / \partial k_{j}\right) / X_{e q}-\left(\partial X_{e q} / \partial k_{j}\right) \cdot X_{i} / X_{e q}^{2}\right\} /\left(\Delta O D_{i} / \Delta O D_{e q}\right)
$$

For the single-step reaction described by equation (5), the partial derivatives of $X(t)$ with respect to the rate constants may be written as

$$
\begin{aligned}
& \partial X / \partial k_{1}=\int_{0}^{t} Z(\tau) Y(\tau) d \tau \\
& \partial X / \partial k_{2}=-\int_{0}^{t} X(\tau) d \tau
\end{aligned}
$$

In this case, the differential equation with the integration may be solved by certain numerical analysis.

(B) Two-step equilibrium reaction :

The differential equations describing the two-step reaction scheme of equation (10) are as follows:

$$
\left\{\begin{array}{l}
d P(t) / d t=-k_{1} P(t)+k_{2} Q(t) \\
d Q(t) / d t=k_{1} P(t)-k_{2} Q(t)-k_{3} Q(t) R(t)+k_{4} S(t) \\
d R(t) / d t=-k_{3} Q(t) R(t)+k_{4} S(t) \\
d S(t) / d t=k_{3} Q(t) R(t)-k_{4} S(t)
\end{array}\right.
$$

where $\mathrm{P}(\mathrm{t})$ is the concentration of $\mathrm{E}_{1}, \mathrm{Q}(\mathrm{t})$ is of $\mathrm{E}_{2}, R(t)$ is of $\mathrm{I}$ and $\boldsymbol{S}(t)$ is of $E_{2}-\mathrm{I}$ complex. The concentration of $E_{2}-I$ complex and the concentration of $E_{2}$ are

$$
S(t)=k_{3} \int_{0}^{t} Q(\tau) R(\tau) d \tau-k_{4} \int_{0}^{t} S(\tau) d \tau
$$

and

$$
Q(t)=k_{1} \int_{0}^{t} P(\tau) d \tau-k_{2} \int_{0}^{t} Q(\tau) d \tau-k_{3} \int_{0}^{t} Q(\tau) R(\tau) d \tau+k_{4} \int_{0}^{t} S(\tau) d \tau
$$


The function $\mathrm{S}(\mathrm{t})$ does not include the $\mathbf{k}$, and $\boldsymbol{k}_{2}$ explicitly. In order to obtain the best fit between the calculated time-course of the concentration of $\mathrm{E}_{2}-\mathrm{I}$ complex and that observed experimentally, one may, therefore, substitute the equation (24) to the function $\mathrm{S}(\mathrm{t})$ of equation (23).

$$
\begin{array}{r}
S(t)=k_{3} \int_{0}^{t}\left(k_{1} \int_{0}^{\tau} P(\xi) d \xi-k_{2} \int_{0}^{\tau} Q(\xi) d \xi-k_{3} \int_{0}^{\tau} Q(\xi) R(\xi) d \xi\right. \\
\left.+k_{4} \int_{0}^{\tau} S(\xi) d \xi\right) R(\tau) d \tau-k_{4} \int_{0}^{t} S(\tau) d \tau
\end{array}
$$

The partial derivatives of the objective function $\mathbf{F}$ with respect to the each rate constant can be calculated by equation (25). When the experimental curve is fitted to the calculated time-course of the concentration of $\mathrm{E}$, , one may directly use the equation (24).

\section{COMPUTATION}

\section{Computation by chemical relaxation method}

The flow chart of the iterative computation is shown in Fig. 6. The rate constans, $k_{1}$ and $k_{2}$, and the equilibrium constant, $\boldsymbol{K}_{1}$, were calculated to be 3.47 $\times 10^{-3} \mathrm{sec}^{-1} \mathrm{M}^{-1}, 3.95 \times 10^{-3} \mathrm{sec}^{-1}$ and $1.138 \mathrm{M}^{-1}$, respectively. The calculated reciprocal relaxation times and equilibrium concentrations are summarized in Table 2. The plotting of the reciprocal relaxation tims $v s$ the sum of the equilibrium concentrations is shown in Fig. 7.

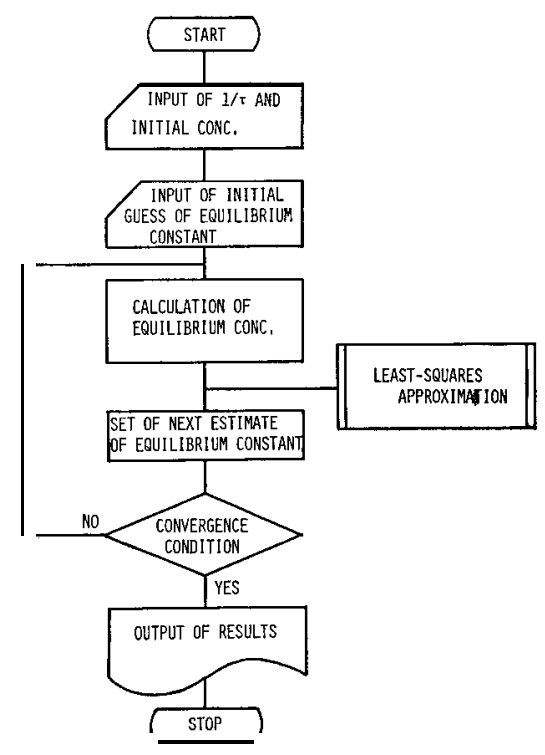

Fig. 6. The flow chart of the iterative computation. 
Table 2. The reciprocal relaxation times and equilibrium concentrations evaluated by the iterative computation.

\begin{tabular}{c|c|c|c}
\hline $\begin{array}{c}\bar{E}^{\mathrm{a})} \\
\left(\times 10^{-4} \mathrm{M}\right)\end{array}$ & $\begin{array}{c}\bar{I}^{\mathrm{b}} \\
\left(\times 10^{-1} \mathrm{M}\right)\end{array}$ & $\begin{array}{c}\bar{E}+\bar{I} \\
\left(X^{-1} \mathrm{M}\right)\end{array}$ & $\begin{array}{c}1 / 0^{-} \\
\left(\times 10^{-2} \sec ^{-1}\right)\end{array}$ \\
\hline 0.6783 & 0.0283 & 0.029 & 0.432 \\
0.6766 & 0.0565 & 0.057 & 0.431 \\
0.6717 & 0.1410 & 0.142 & 0.377 \\
0.6700 & 0.1690 & 0.170 & 0.396 \\
0.6668 & 0.2250 & 0.226 & 0.367 \\
0.6636 & 0.2820 & 0.283 & 0.306 \\
0.6478 & 0.5650 & 0.566 & 0.503 \\
0.6329 & 0.8460 & 0.847 & 0.444 \\
0.6186 & 1.130 & 1.131 & 0.459 \\
& 1.410 & 1.411 & 0.403 \\
\hline
\end{tabular}

a) Equilibrium concentration of lysozyme.

b) Equilibrium concentration of DBMA.

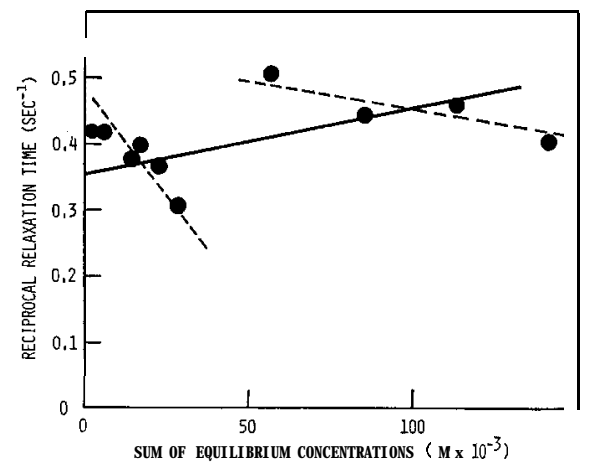

Fig. 7. The reciprocal relaxation time as a function of the sum of the equilibrium concentration of lysozyme and DBMA. A solid line was estimated by the iterative computation.

The flow chart of the nonlinear least-squares method is shown in Fig. 8. The inverse matrix was evaluated by the FACOM 230-60 Scientific Subroutine Library (ID code : G/002/D). The partial derivatives of the objective function with respect to the rate constants were obtained analytically. However, the convergence of the optimization was not fully attained.

\section{Computation by direct curve-fitting method}

The flow chart of the direct curve-fitting method is shown in Fig. 9. The simultaneous ordinary differential equation was solved numerically by the RungeKutta-Gill method using the FACOM 230-60 Scientific Subroutine Library (ID code $: F / 002 / D)$. In the algorithm of the FR method, the step c) for the linear search was omitted to cut down the CPU time for the computation and the value of a was fixed to $\mathbf{0 . 1}$ arbitrarily. When the value of a was fixed to 1.0, the value of the objective function tended to divergence. To obtain the stability of the Runge-Kutta-Gill method, the real time-scale was converted into an appropriate scale. For the value of rate constants, there must be the nonnega- 


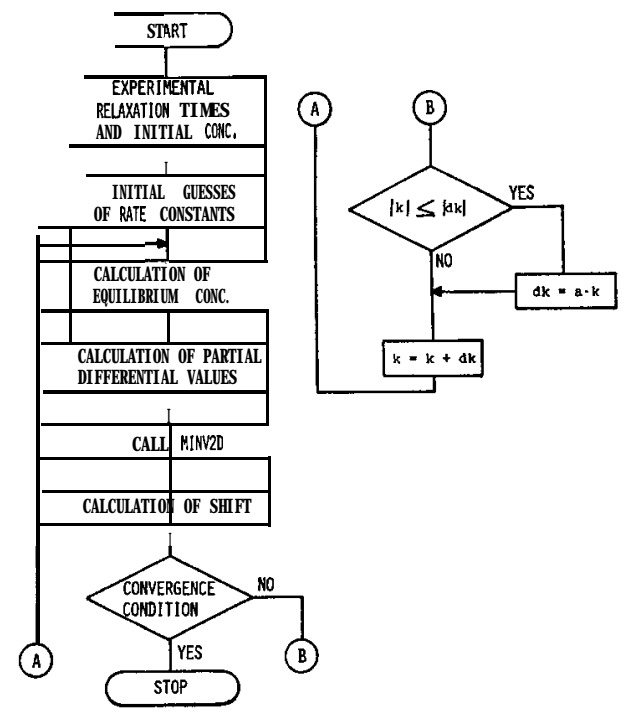

Fig. 8. The flow chart of the nonlinear least-squares method. MINV2D is the subprogram for the calculation of inverse matrix.

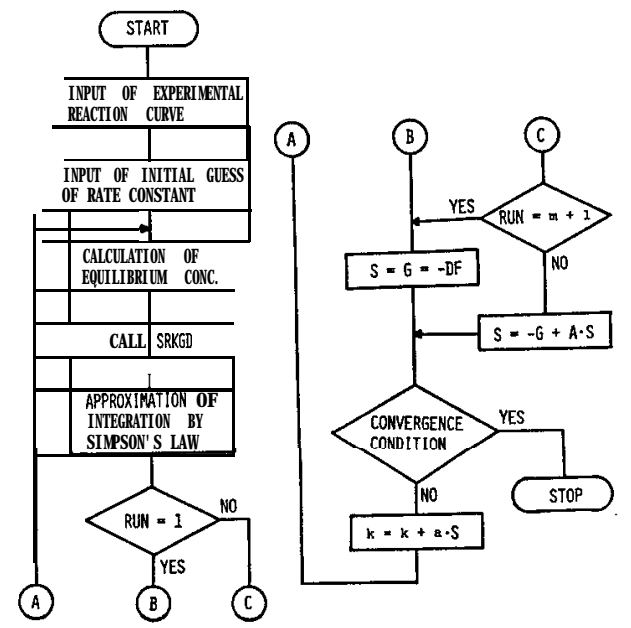

Fig. 9. The flow chart of the direct curve-fitting method by the FR method. SRKGD is the subprogram for the calculation of differential equation.

tive constraint condition. The authors first made use of the penalty function method to receive this constraint condition. However, better results were obtained by the simple adjustment of the value of a in each iteration step. On the accuracy of the computation, it was observed that the value of the equilibrium concentrations had often became negative and the optimization had diverged. This was mainly due to the inadequate selection of values of the initial guess for parameters. Such behaviors in computation can, therefore, be prevented by simple 
caution to use the suitable initial guess. Table 3 summarizes the finally estimated rate constants. The time-course of the chemical species calculated by the estimated rate constants are shown in from Fig. 10 to Fig. 19.

Table 3. The estimated rate constants by the direct curve-fitting method.

\begin{tabular}{|c|c|c|c|c|c|}
\hline & & $k_{1}\left(\sec ^{-1}\right)$ & $k_{2}\left(\sec ^{-1}\right)$ & $k_{3}\left(\sec ^{-1} M^{-1}\right)$ & $k_{4}\left(\sec ^{-1}\right)$ \\
\hline \multirow[b]{2}{*}{ (1) } & Single-step & $0.1034 \times 10^{-1}$ & 0 & $5722 \times 10^{-2}$ & \\
\hline & $\frac{\left.E_{2} I^{a}\right)}{\left.E_{2}\right)}$ & $\begin{array}{l}0.2004 \\
0.5362 \times 10^{-2}\end{array}$ & $\begin{array}{l}0.1706 \times 10-r \\
0.5360 \times 10^{-3}\end{array}$ & $\begin{array}{l}0.1999 \\
0.2000\end{array}$ & $\begin{array}{l}0.5277 \times 10^{-2} \\
0.2005 \times 10^{-1}\end{array}$ \\
\hline (2) & $\frac{\mathrm{E}_{2} \mathrm{I}}{\mathrm{E}_{2}}$ & $\begin{array}{l}0.2017 \\
0.7444 \times 10-2\end{array}$ & $\begin{array}{l}0.3885 \times 10^{-2} \\
0.4675 \times 10^{-3}\end{array}$ & $\begin{array}{l}0.2003 \\
0.1997 \times 10^{-1}\end{array}$ & $\begin{array}{l}0.7078 \times 10^{-2} \\
0.2222 \times 10-2\end{array}$ \\
\hline \multirow[b]{2}{*}{ (3) } & Single-step & $0.4987 X 10-r$ & $0.5913 \times 10-$ & 2 & \\
\hline & $\begin{array}{l}\mathrm{E}_{2} \mathrm{I} \\
\mathrm{E}_{2}\end{array}$ & $\begin{array}{l}0.3890 \\
0.8073 \times 10^{-3}\end{array}$ & $\begin{array}{l}0.4112 \\
0.7022 X 10-\mathrm{r}\end{array}$ & $\begin{array}{l}0.4964 \\
0.5000\end{array}$ & $\begin{array}{l}0.5756 \times 10^{-2} \\
0.4991+10^{-1}\end{array}$ \\
\hline (4) & $\frac{\mathrm{E}_{2} \mathrm{I}}{\mathrm{E}_{2}}$ & $\begin{array}{l}0.5002 \\
0.5353 \times 10-3\end{array}$ & $\begin{array}{l}0.4898 \times 10^{-1} \\
0.9198 \times 10^{-2}\end{array}$ & $\begin{array}{l}0.5000 \\
0.5000\end{array}$ & $\begin{array}{l}0.9608 \times 10^{-2} \\
0.4995 \times 10^{-1}\end{array}$ \\
\hline
\end{tabular}

The concentrations of lysozyme were $0.680 \times 10^{-4} \mathrm{M}$ for (1) and (2) ; and $0.105 \times 10^{-3} \mathrm{M}$ for (3) and (4). The concentrations of DBMA were $0.280 \times 10^{-2} \mathrm{M}$ for (1), $0.226 \times 10^{-2}$ $\mathrm{M}$ for (2), $0.141 \times 10^{-2} \mathrm{M}$ for (3) and $0.847 \times 10^{-3} \mathrm{M}$ for (4). The two-step reaction scheme was used for the time-course of $\left.E_{2}-I(a)\right)$ and that of $E_{2}(b)$ ) to be fitted to the experimental time-course.

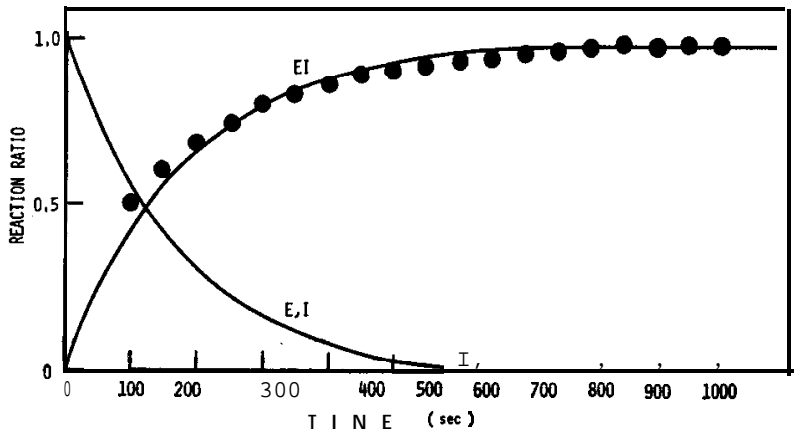

Fig. 10. The calculated curves for the single-step reaction scheme. The initial concentrations and the estimated rate constants are listed in (1) at Table 3. (O):Experimental data points.

\section{DISCUSSION}

It has been observed that, under the fixed concentration of lysozyme, the equilibrating reaction rate, hence the reciprocal relaxation time, decreased with the increase in the DBMA concentration (Hayashi et al., 1968a). This fact indicated that the lysozyme-inhibitor interaction would proceed along a complex scheme. As expected, the single-step reaction scheme represented by equation (5) failed to explain the lysozyme-inhibitor interaction, i. e., the estimated values 


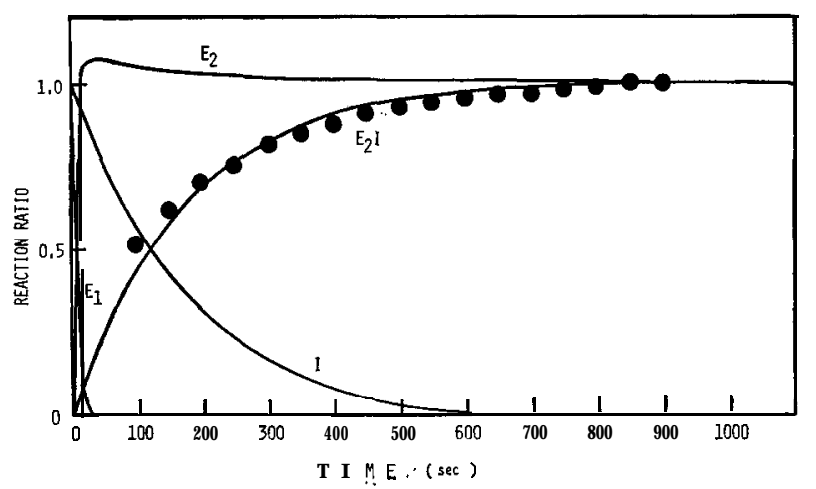

Fig. 11. The calculated curves to be fitted to the time-course of $E_{2}-I$ for the two-step reaction scheme. The initial concentrations and the estimated rate constants are listed in (1) at Table 3. (0): Experimental data points.

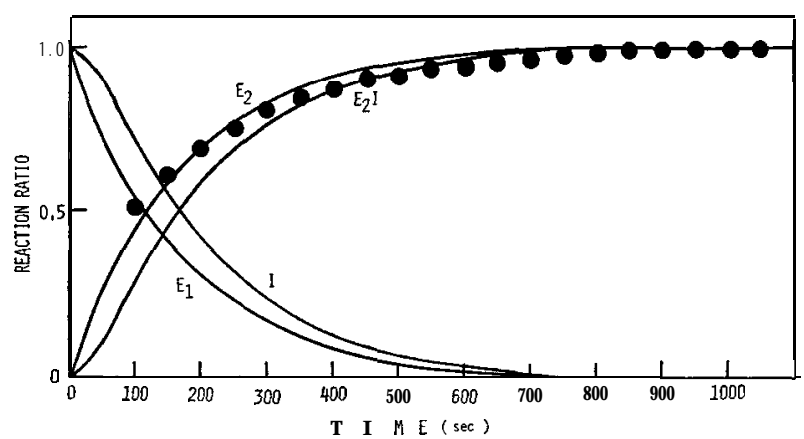

Fig. 12. The calculated curves to be fitted to the time-course of $E_{2}$ for the two-step reaction scheme. The initial concentrations and the estimated rate constants are listed in (1) at Table 3. ( ) : Experimental data points.

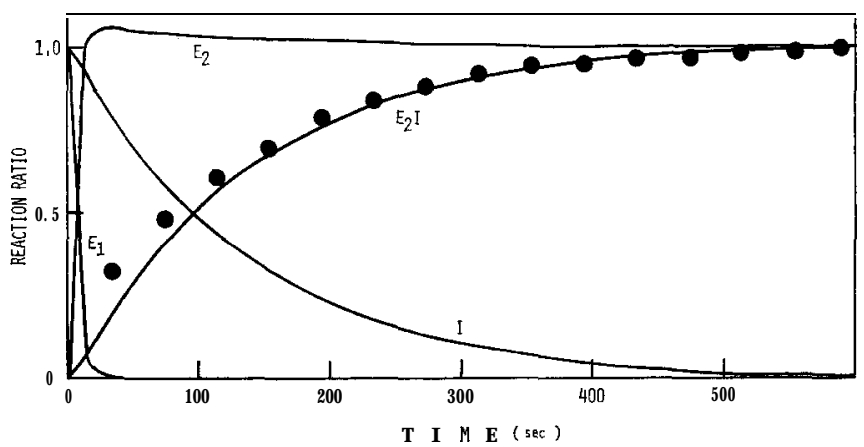

Fig. 13. The calculated curves to be fitted to the time-course of $E_{2}-\mathrm{I}$ for the two-step reaction scheme. The initial concentrations and the estimated rate constants are listed in (2) at Table 3. (0): Experimental data points. 


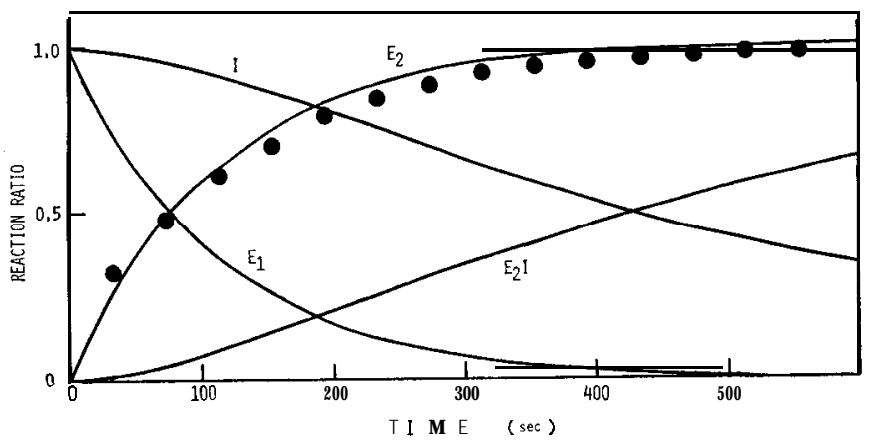

Fig. 14. The calculated curves to be fitted to the time-course of $E_{2}$ for the two-step reaction scheme. The initial concentrations and the estimated rate constants are listed in (2) at Table 3. (0): Experimental data points.

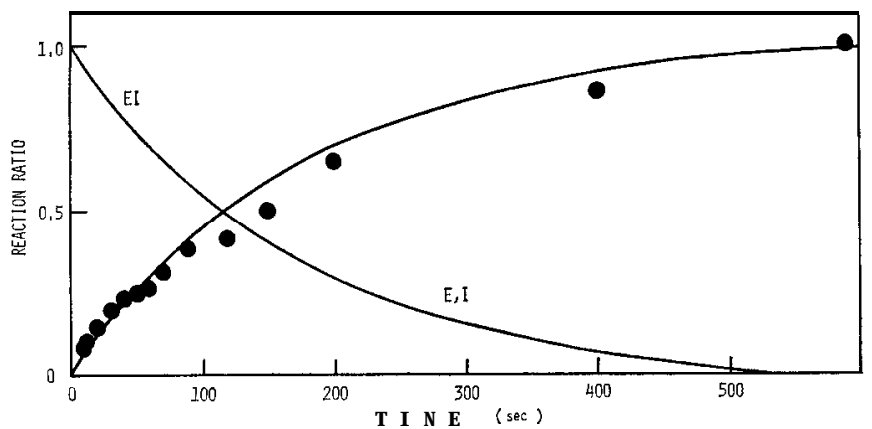

Fig. 15. The calculated curves for the single-step reaction scheme. The initial concentrations and the estimated rate constants are listed in (3) at Table 3. ( ) : Experimental data points.

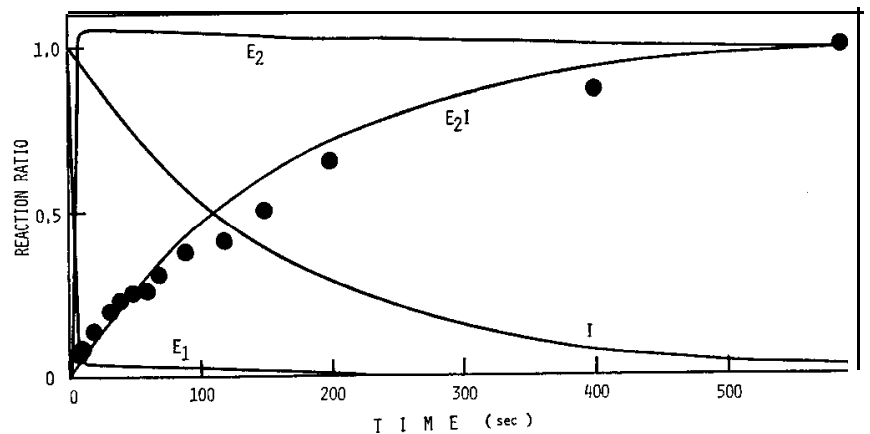

Fig. 16. The calculated curves to be fitted to the time-course of $E_{2}-I$ for the two-step reaction scheme. The initial concentrations and the estimated rate constants are listed in (3) at Table 3. (๑): Experimental data points. 


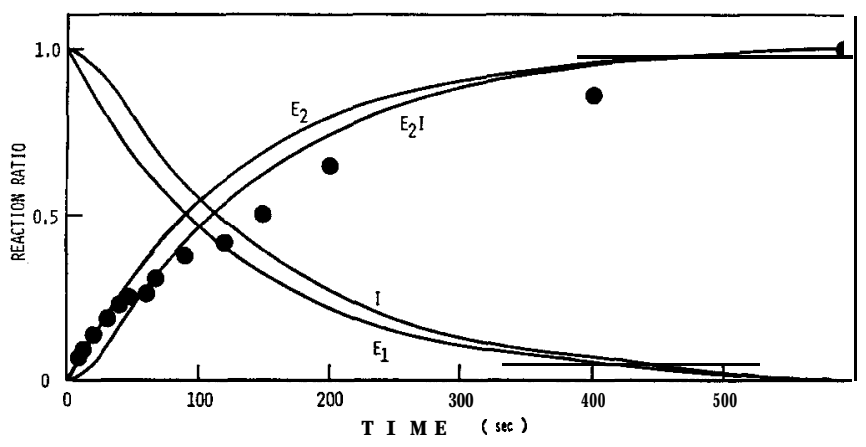

Fig. 17. The calculated curves to be fitted to the time-course of $\mathrm{E}_{2}$ for the two-step reaction scheme. The initial concentrations and the estimated rate constants are listed in (3) at Table 3. (•): Experimental data points.

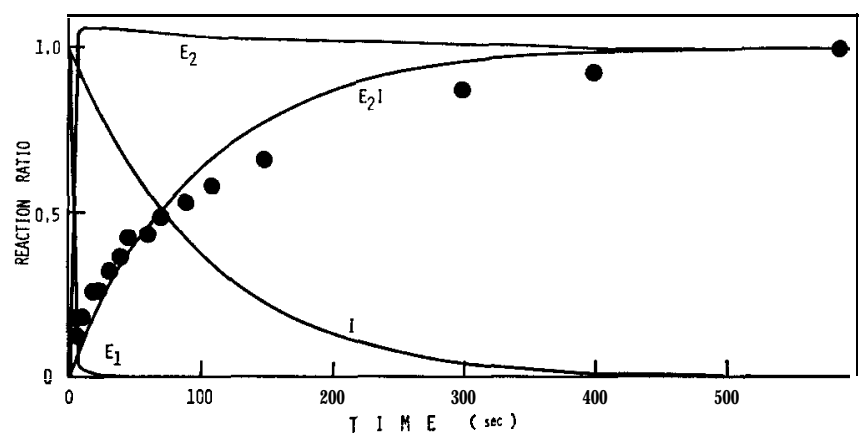

Fig. 18. The calculated curves to be fitted to the time-course of $E_{2}-I$ for the two-step reaction scheme. The initial concentrations and the estimated rate constants are listed in (4) at Table 3. (0): Experimental data points.

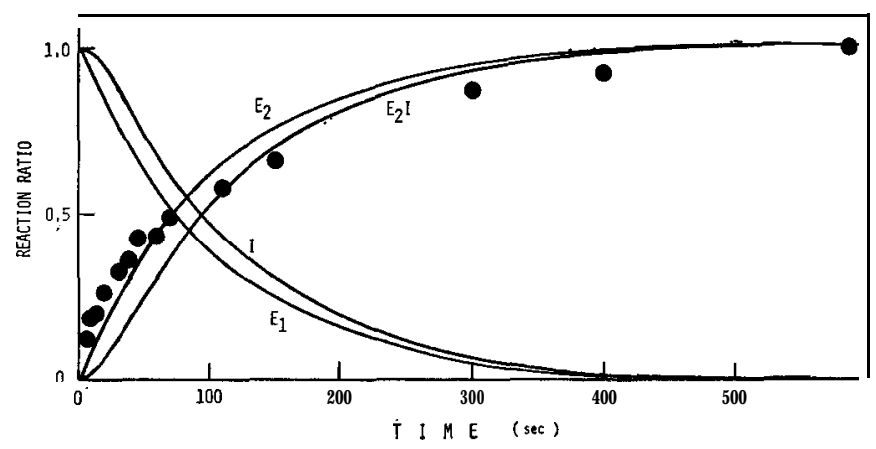

Fig. 19. The calculated curves to be fitted to the time-course of $E_{2}$ for the two-step reaction scheme. The initial concentrations and the estimated rate constants are listed in (4) at Table 3. (0): Experimental data points. 
of the rate constants by the iterative computation were not reasonable. Consequently, the authors assumed two-step reaction scheme represented by equation (10). The optimization methods were adopted to estimate the rate constants. One is the nonlinear least-squares method which is derived in order to fit the calculated relaxation time to experimentally obtained one, and the other the FR method which is based upon the direct comparison of the calculated time-course of the reaction with the experimentally obtained time-course. The former method did not give a satisfactory result because of either application of unreasonable approximation for chemical relaxation system or the instability of the program for the computation.

The FR method gave the reasonable estimate of the rate constants, suggesting that this method may be applied inclusively for the present and similar kinds of the experiments. When the initial concentration of the inhibitor was varied under the estimated rate constants in Fig. 11, the calculated time-course of $\mathrm{E}_{2}-\mathrm{I}$ complex was slightly altered as shown in Fig. 20. This was not in agreement with the experimental results. On the other hand the calulated time-course of $\mathrm{E}_{2}$ rised once over the corresponding equilibrium concentration and then reach to the equilibrium state as shown in Fig. 21. The degree of such deviation from the equilibrium state increased as the initial concentration of the inhibitor was increased. From these facts, it was assumed that the time-course of $\Delta \mathrm{OD}_{296}$ corresponds to that of $\mathrm{E}_{2}$.

In the previous study, Hayashi et al. (196813) found by the equilibrium dialysis that six molecules of DBMA bound with one molecule of lysozyme. According to that finding and the present results, it is likely that the following reaction scheme may be possible under limited range of the initial concentration of DBMA, and the number of DBMA molecule per molecule of lysozyme may be sensitive for the variation of the initial concentration of the inhibitor.

$$
\mathrm{E}_{1} \rightleftarrows \mathrm{E}_{2}+61 \rightleftarrows \mathrm{E}_{2}-\mathrm{I}_{6}
$$

The pH-dependence of the inhibitory action of DBMA against the activity of lysozyme showed the inverse pattern of pa-activity profile for the untreated lysozyme. This fact seems to be interpreted qualitatively by the variation of

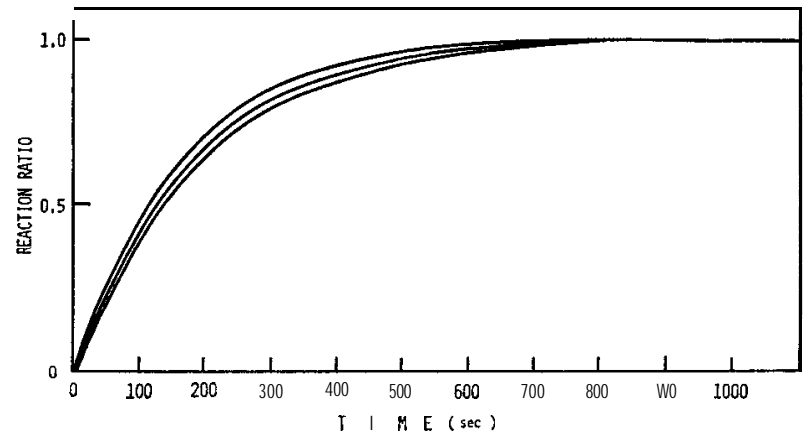

Fig. 20. The calculated time-course of $E_{2}-I$ as a function of initial concentration of DBMA. The concentrations of DBMA from top to bottom were $0.00564 \mathrm{M}, 0.00282 \mathrm{M}$ and $0.00141 \mathrm{M}$. The fixed rate constants are listed in (1) at Table 3. 


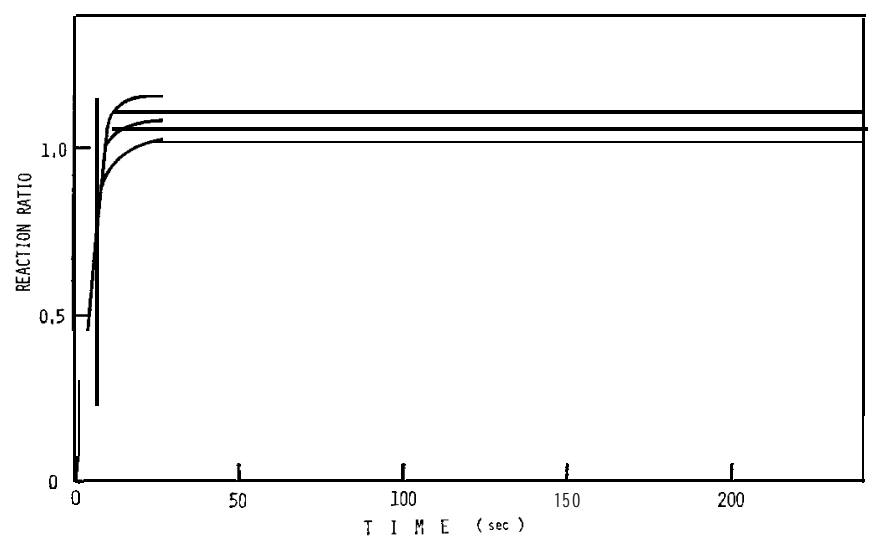

Fig. 21. The calculated time-course of $\mathrm{E}_{2}$ as a function of initial concentration of DBMA. The concentrations of DBMA from top to bottom were $0.00564 \mathrm{M}, 0.00282 \mathrm{M}$ and $0.00141 \mathrm{M}$. The fixed rate constants are listed in (1) at Table 3.

the equilibrium constant between $E_{1}$ and $E_{2}$, if it is assumed that $E_{1}$ is active for the substrate and $\mathrm{E}_{2}$ is inactive.

Thus, the scheme represented by equation (10) may be reasonable candidate for the equilibrating reaction in lysozyme-DBMA interaction system.

\section{REFERENCES}

Castellan, G. W. 1963 Calculation of Spectrum of Chemical Relaxation Times for a General Reaction Mechanism. Ber. Bunsenges. Physik. Chem., 67: 898-908

Duynstee, E. F. and E. Grunwald 1959 Organic Reactions Occuring in or on Micelles. I. Reaction Rate Studies of Alkaline Fading of Triphenylmethane Dyes and Sulfonphthalein Indicators in Presence of Detergent Salts. J. Am. Chem. Soc., 81: 4550-4542

Eigen, M. and L. DeMaeyer 1963 Relaxation Methods In "Technique of Organic Chemistry" Vol. III part II ed. by S. L. Friess, L. S. Lewis and A. Weissberger, Interscience Pub. Inc., New York, pp 895

Hayashi, K., T. Imoto and M. Funatsu 1963 The Enzyme-substrate Complex in a Muramidase Catalyzed Reaction. J. Biochem. (Tokyo), 54: 381-387

Hayashi, K., M. Kugimiya, T. Imoto, M. Funatsu and C. C. Bigelow 1968a The Inhibitory Interaction of Cationic Detergents with the Active Center of Lysozyme. I. Site of Interaction. Biochemistry, $7:$ 1461-1466

Hayashi, K., M. Kugimiya, T. Imoto, M. Funatsu and C. C. Bigelow 1968b The Inhibitory Interaction of Cationic Detergents with the Active Center of Lysozyme. II. The pH Dependence of the Interaction. Biochemistry, 7: 1467-1472

Sharma, V. S. and D. L. Leussing 1971 A General Computer Approach for Calculating Rate Constants from Near-equilibrium Kinetic Studies. Talanta, 18:1137-1155 